\title{
Flight Test Results of a GPS-Based Pitot-Static Calibration Method Using Output-Error Optimization for a Light Twin- Engine Airplane
}

\author{
Borja Martos ${ }^{1}$ and Paul Kiszely ${ }^{2}$ \\ University of Tennessee Space Institute, Tullahoma, TN 37388 \\ and \\ John V. Foster ${ }^{3}$ \\ NASA Langley Research Center, Hampton, VA 23681
}

\begin{abstract}
As part of the NASA Aviation Safety Program (AvSP), a novel pitot-static calibration method was developed to allow rapid in-flight calibration for subscale aircraft while flying within confined test areas. This approach uses Global Positioning System (GPS) technology coupled with modern system identification methods that rapidly computes optimal pressure error models over a range of airspeed with defined confidence bounds. This method has been demonstrated in subscale flight tests and has shown small 2-o error bounds with significant reduction in test time compared to other methods. The current research was motivated by the desire to further evaluate and develop this method for full-scale aircraft. A goal of this research was to develop an accurate calibration method that enables reductions in test equipment and flight time, thus reducing costs. The approach involved analysis of data acquisition requirements, development of efficient flight patterns, and analysis of pressure error models based on system identification methods. Flight tests were conducted at The University of Tennessee Space Institute (UTSI) utilizing an instrumented Piper Navajo research aircraft. In addition, the UTSI engineering flight simulator was used to investigate test maneuver requirements and handling qualities issues associated with this technique. This paper provides a summary of piloted simulation and flight test results that illustrates the performance and capabilities of the NASA calibration method. Discussion of maneuver requirements and data analysis methods is included as well as recommendations for piloting technique.
\end{abstract}

\section{Nomenclature}

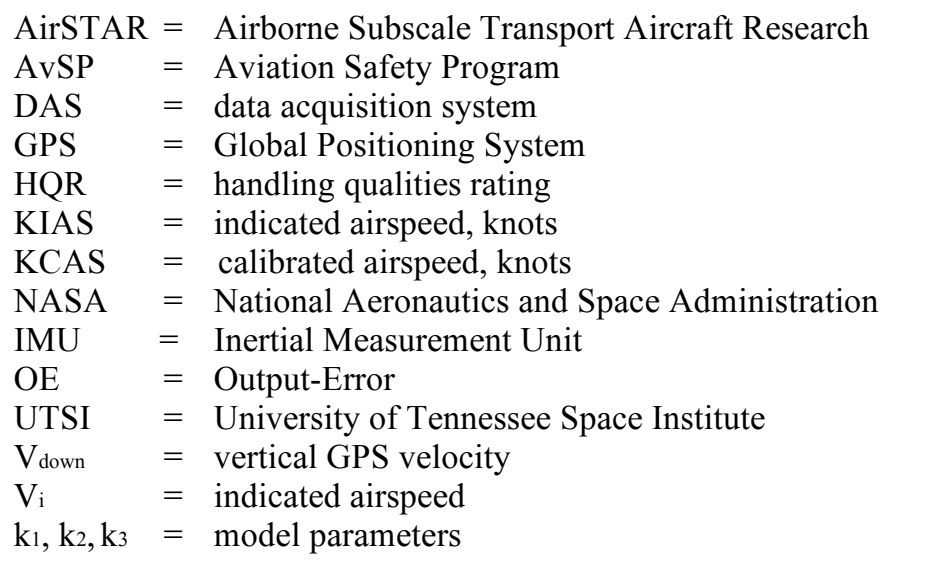

${ }^{1}$ Research Engineer, Aviation Systems \& Flight Research, MS 20, AIAA Member.

${ }^{2}$ CW4, U.S.Army, Graduate Student, Aviation Systems \& Flight Research, MS 20.

${ }^{3}$ Senior Research Engineer, Flight Dynamics Branch, MS 308, AIAA Associate Fellow. 


$\begin{array}{ll}\mathrm{q}_{\mathrm{c}} & =\text { impact pressure } \\ \mathrm{q}_{\mathrm{ci}} & =\text { indicated impact pressure } \\ \Delta \mathrm{p} & =\text { pressure error, } \mathrm{q}_{\mathrm{c}}-\mathrm{q}_{\mathrm{ci}} \\ \sigma & =\text { standard deviation }\end{array}$

\section{Introduction}

Dressure-based airspeed and altitude measurements for aircraft typically require calibration of the installed system to account for pressure sensing errors such as those due to local flow field effects. Various methods are used for in-flight pitot-static calibration including tower fly-by, pacer aircraft, and trailing cone methods. In the 1990's, the introduction of satellite-based positioning systems to the civilian market enabled new inflight calibration methods based on accurate ground speed measurements provided by Global Positioning Systems (GPS). Several GPS-based airspeed calibration methods are currently used such as the 'three-leg' and 'four-leg' methods ${ }^{1}$. As part of the NASA Aviation Safety Program (AvSP), a novel GPS-based method was developed to allow rapid in-flight calibration for subscale aircraft while flying within confined test areas ${ }^{2}$. This approach uses system identification methods that rapidly compute optimal pressure error models over a range of airspeed with defined confidence intervals. This method has been demonstrated in sub-scale flight tests and has shown small 2- $\sigma$ error bounds with significant reduction in test time over other GPS methods.

The current research was motivated by the desire to further evaluate and develop the NASA GPS-based calibration method for full-scale aircraft. A goal of this research was to develop an accurate technique that enables reductions in test equipment and flight time, thus reducing costs. The approach involved analysis of data acquisition requirements, development of optimal flight patterns, and analysis of pressure error models based on system identification methods. Flight tests were conducted at The University of Tennessee Space Institute (UTSI), utilizing an instrumented Piper Navajo research aircraft. In addition, the UTSI engineering flight simulator was used to investigate test maneuver requirements and handling qualities issues associated with this technique in support of the flight tests.

\section{Method of Tests}

The objective of this research was to evaluate and further develop the NASA GPS-based calibration method for full scale applications using an existing flight test aircraft. The key elements of this research included: 1) flight maneuvers and patterns for data acquisition including pilot workload assessments, 2) data requirements such as sample rate, maneuver length, and sensor measurement accuracy, 3) data reduction methods and calculation of confidence bounds, and 4) interpretation of results and practical application in a full scale flight test environment. As part of this effort, consideration for the underlying theory and assumptions was reviewed and the method was compared to other existing GPS-based approaches.

Specific goals of this research included the following.

- Recommendations for calibration maneuvers based on pilot workload, test time requirements, and data accuracy

- Recommendations and strategies for identification of calibration models

- Quantified effects of data acquisition system characteristics, maneuver pattern, and atmospheric conditions (wind variations and turbulence) on calibration accuracy

- Recommendations for test techniques and data reduction method that can potentially reduce test time and complexity

\section{A. Description of Calibration Method}

The calibration method used is a GPS-based approach that was developed as part of the NASA AirSTAR subscale flight test facility and this method is described in Reference 2. The method used system identification algorithms to compute a continuous calibration model as a function of indicated airspeed, rather than for fixed airspeed conditions, and it provided statistical boundaries to assess the calibration accuracy. The method has demonstrated several advantages over other methods including reduced flight test time, less restricted maneuver requirements, and statistical control over calibration accuracy.

The optimization algorithm used for this research was from the System IDentification Programs for AirCraft (SIDPAC) toolbox (described in Reference 3) for MATLAB ${ }^{\circledR}$. The output error routine (OE) was used as the primary tool for identification of model parameters. The output-error method computed pre-defined model parameters based on minimization of error between test data and model predictions. The strategy involved resolution of velocity vectors, namely ground speed, true airspeed, and wind speed. For this application, the calibration data were estimates of normalized pressure error as a function of impact pressure (discussed in a later section). The 
model parameters were equation coefficients for normalized pressure error and the wind vector velocity and direction.

For this application, the underlying assumptions were critical for evaluation and practical implementation of this method. First, it was assumed the wind vector was constant (without a vertical component for some cases) for the entire calibration maneuver, and the algorithm computed the best estimate of the wind direction and velocity. Therefore all calibration maneuvers were flown at constant altitude to minimize the wind variability. It can be asserted that wind variability may also be reduced by conducting the calibration maneuver in minimum time. Secondly, the algorithm assumes that ground speed was the sum of true airspeed and wind speed which only holds true for steady flight conditions. While most existing calibration methods are conducted at steady airspeed and heading, this study addressed the potential to include quasi-steady conditions, such as slowly varying airspeeds and slow turns, as a way of acquiring data more rapidly without sacrificing calibration accuracy.

\section{B. Description of Test Aircraft}

The test aircraft used for this research was a PA-31 Navajo (Fig. 1), a cabin-class, twin-engine aircraft designed and built by Piper Aircraft, Inc. for the general aviation market. The aircraft has a wingspan of $40.67 \mathrm{ft}(12.40 \mathrm{~m})$, fuselage length of $32.63 \mathrm{ft}(9.94 \mathrm{~m})$, and a maximum gross weight of $6,500 \mathrm{lbs}(2,948 \mathrm{~kg})$. The UTSI Navajo can carry a maximum crew of six, nominally two pilots in the cockpit and four aircrew or passengers in the main cabin.

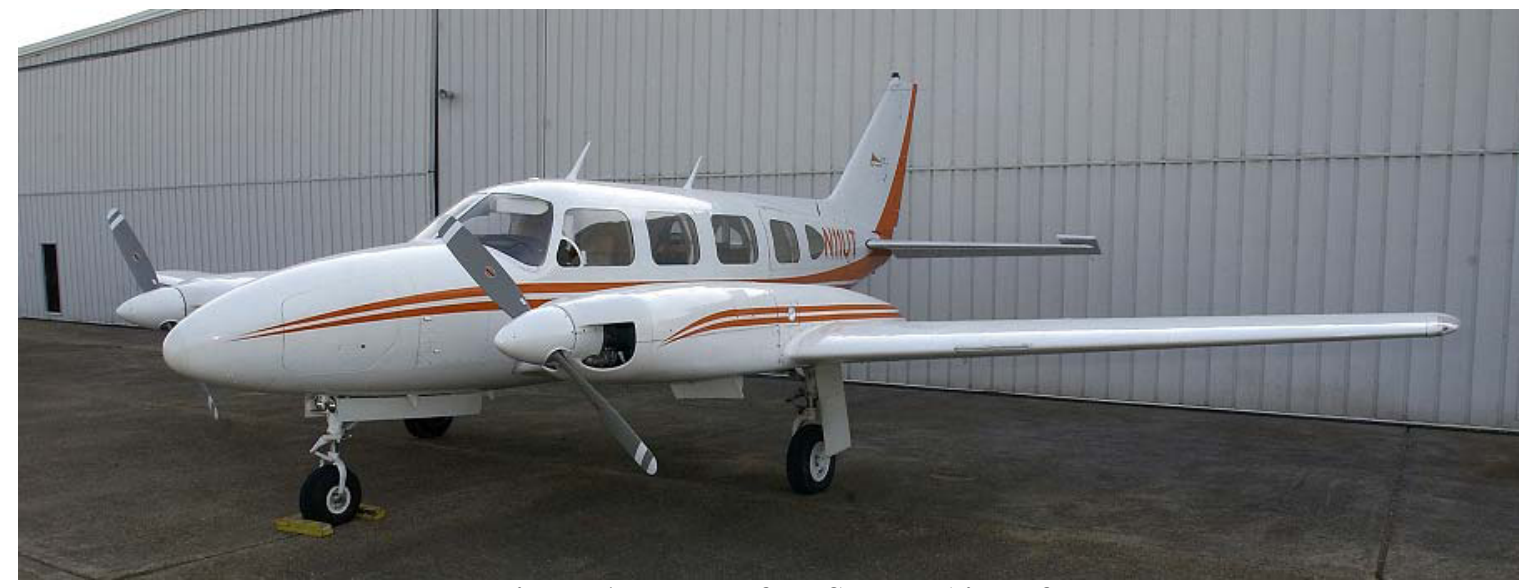

Figure 1. Photo of UTSI Test Aircraft.

The Navajo is powered by two turbocharged Lycoming TIO-540-A series, six cylinder, fuel injected engines rated at 310 horsepower each. An oxygen system provides supplementary oxygen for crew and passengers. The aircraft has a service ceiling of $26,300 \mathrm{ft}(8,016.2 \mathrm{~m})$. During the data flights the Navajo was outfitted with an instrumentation belly pod not shown in Fig. 1.

The research aircraft has two air data systems. The research air data system is independent of the production air data system. The research system includes a Kiel probe, a standard total pressure probe, a static pressure mast, and a total temperature probe. The probes are mounted on the left fuselage, forward of the wing and aft of the nose baggage compartment door. The data acquisition system (DAS) records air data parameters at twenty samples per second with the exception of the GPS signal which is logged at one sample per second.

\section{Description of Engineering Simulator}

The UTSI engineering simulator, shown in Fig. 2, integrates simulation models with commercial off-the-shelf and custom hardware for pilot-in-the-loop evaluations. The simulation models are hosted by MATLAB SIMULINK ${ }^{\circ}$ software on PC-based computers and the visual scenery is generated by X-Plane ${ }^{\circledR}$. The simulation models use state and pilot control inputs to calculate force and moments in real time, provide outputs from the equations of motion, and update cockpit and out-the-window visuals to the pilot. The simulator uses three 62 -inch displays for out-the-window visuals, three flat panel touch screens inside the cockpit, and representative pilot controls. A total of seven PC computers are used in real time. Three computers generate out-the-window visuals, two computers generate cockpit instrumentation and research displays, one computer serves as the flight director's ground station, and one computer runs the simulation models in hard real-time. 


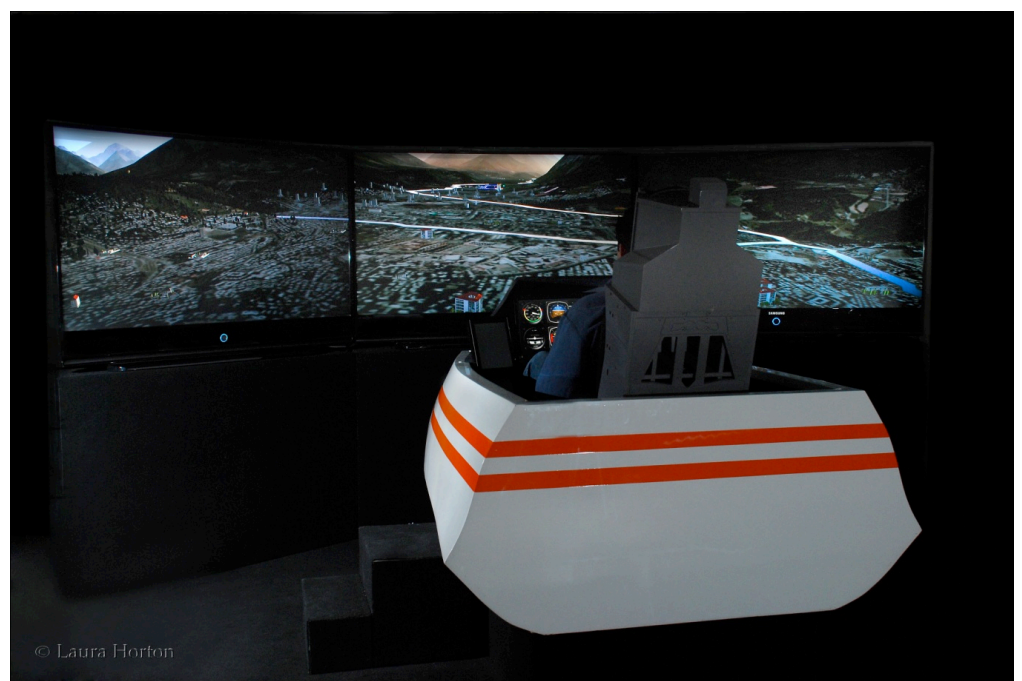

Figure 2. Photo of UTSI Engineering Flight Simulator.

\section{Results and Discussion}

\section{A. Description and Evaluation of Calibration Maneuvers}

Five calibration maneuvers, illustrated in Fig. 3, were evaluated in this research. Each maneuver was selected based on the potential to rapidly acquire data in a small test area while providing maximum time for steady flight conditions. Four of these maneuvers were evaluated in the UTSI Piper Navajo: 4-Leg, Race Track, Step Decrease and Rapid Deceleration (labeled as "Rapid Decel"). The simulator was also used to evaluate these four maneuvers, and in addition the Circle maneuver was evaluated as an alternative approach for comparison and handling qualities assessment.

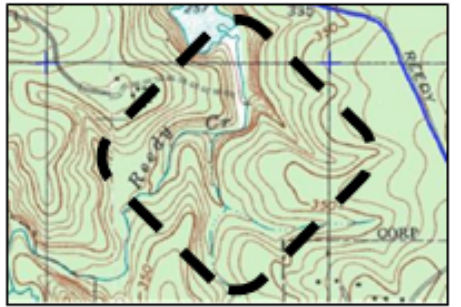

GPS-4-Leg

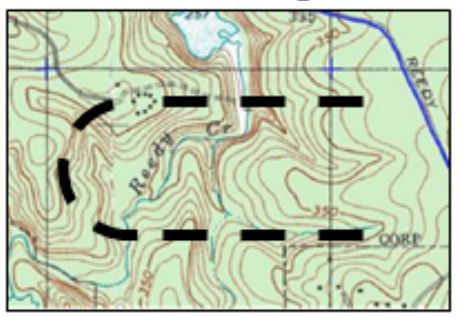

Step Decrease/Rapid Decel

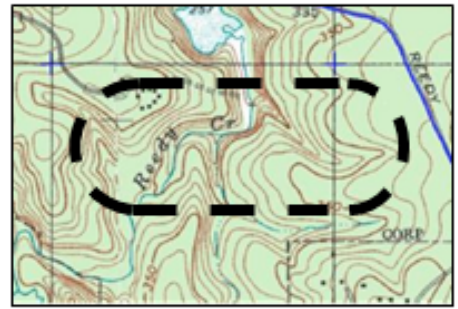

Race Track

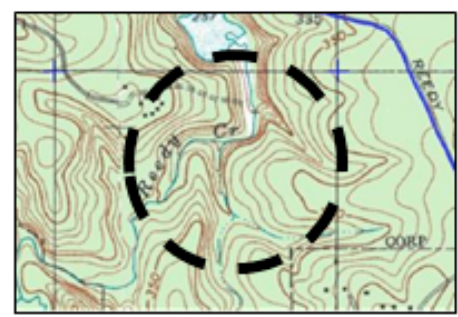

Circle

Figure 3. Illustration of Calibration Maneuvers (shown for no wind conditions).

The 4-Leg maneuver (sometimes referred to as the "GPS-4-leg") was flown with straight legs, 90 degrees apart in heading, and between 5 and 10 seconds of on-condition data capture for each leg. A constant target airspeed was flown for each complete circuit and repeated for each additional airspeed. Aircraft and simulator flights consisted of four to six different airspeeds. Maneuver tolerances were defined as +/-1 KCAS for airspeed, +/-2 
degrees for heading, and +/- 50 feet for altitude. Since the 4-Leg method is designed for steady flight conditions, maintaining airspeed and heading during this maneuver were the primary objectives while altitude was the secondary objective. Airspeeds used for this maneuver were 160, 140, 120, and 100 KCAS and each 4-Leg circuit was completed in approximately 5-7 minutes.

The Race Track maneuver was flown at a constant target airspeed on reciprocal headings for one circuit. Oncondition data capture time was 60 seconds per straight leg portion. As with the 4-Leg method, four different airspeeds were flown with the same tolerances where airspeed and heading tolerances were the primary objectives. Airspeeds used for this test were 160, 140, 120, and 100 knots. Each racetrack circuit was completed in approximately 3-4 minutes.

The Step Decrease maneuver started at $160 \mathrm{KCAS}$, and a power reduction of one inch of manifold pressure was applied every 5-10 seconds until slowing to $130 \mathrm{KCAS}$ along one heading. A turn was made to the reciprocal heading, and the same power reductions were applied until reaching $100 \mathrm{KCAS}$. There was no airspeed tolerance for this maneuver since it is a gradual speed reduction throughout. Heading was maintained within $+/-2$ degrees and altitude within $+/-50$ feet. The maneuver was completed in approximately 5-6 minutes.

The Rapid Deceleration maneuver started at $160 \mathrm{KCAS}$ with power then reduced to idle and the aircraft allowed to slow to $100 \mathrm{KCAS}$. Altitude was maintained within $+/-50$ feet and heading of $+/-2$ degrees along one heading. A turn was made to the reciprocal heading and the maneuver repeated. Approximate time for this maneuver was under 5 minutes.

The Circle maneuver was only tested in the simulator and several bank angles were evaluated to optimize the handling qualities of the maneuver. Ten degrees of bank proved to be difficult to maintain for the length of time needed to complete the Circle. This was a very shallow bank angle and tedious to hold. A 20 degree bank angle manifested much of the same characteristics as with 10 degrees of bank. The scaling on the attitude indicator did not have a reference for 45 degrees, so holding that constant bank angle was not possible. This bank angle also required addition of power in the maneuver to maintain airspeed and altitude adding a degree of difficulty. With 30 degrees of bank angle, airspeed and altitude could be maintained with the power that was used to enter the maneuver. Holding this bank angle was not difficult, and the time to complete the Circle was small, therefore a 30 degree bank angle was selected for this method. Each circular pattern was flown at a constant target airspeed with tolerances of $+/-2$ KCAS and altitude of $+/-50$ feet. The maneuvers were flown at 160, 140, 120, and $100 \mathrm{KCAS}$.

Composite handling qualities ratings (HQR) were provided for all maneuvers evaluated in the Piper Navajo and simulator, and results are shown in Table 1 . The Cooper-Harper rating scale ${ }^{4}$ was used for these assessments where the desired performance criteria were as previously described. The aircraft and simulator tasks were each evaluated by a different pilot, and, therefore, these ratings only represent the opinion of one pilot.

Table 1. Cooper-Harper Pilot Ratings For Calibration Maneuvers.

\begin{tabular}{|ccc|}
\hline Flight Test & $\begin{array}{c}\text { Flight } \\
\text { Technique }\end{array}$ & $\begin{array}{c}\text { Rimulator } \\
\text { Rating }\end{array}$ \\
\hline Step Decrease & $\mathbf{4}$ & $\mathbf{3}$ \\
\hline Race Track & $\mathbf{2}$ & $\mathbf{2}$ \\
\hline GPS-4-Leg & $\mathbf{4}$ & $\mathbf{3}$ \\
\hline Circle & Not Flown & $\mathbf{4}$ \\
\hline Rapid Decel & $\mathbf{2}$ & $\mathbf{2}$ \\
\hline
\end{tabular}

The pilot workload in the aircraft was found to be the highest (Level II) for the Step Decrease and 4-Leg maneuvers. The HQR for the 4-Leg maneuver reflects the pilot compensation required to maintain the defined airspeed, altitude, and heading tolerances for tens of seconds. This is representative of how this maneuver is currently flown in FAA Part 23 flight testing. Likewise the HQR for the Step Decrease maneuver suggests that there were minor but annoying deficiencies. In this particular case, workload was impacted by the placement of the manifold pressure gauges in the cockpit. The manifold pressure gauges were installed directly above the throttle quadrant and are removed from the instrument cluster. Due to the repeated small throttle steps during this maneuver, this arrangement constantly disrupts the pilot's instrument scan. Much of the workload in the Step Decrease maneuver can be alleviated through a co-pilot or through more modern instrument panel layouts. The HQR for the Rapid Deceleration and Race Track maneuvers suggests good (Level I) handling qualities. In general, the 4-Leg 
maneuver had the highest workload due to the duration of the test and to tight tolerances. The significance of these HQRs is best understood in the context of the results discussion.

Based on the simulator evaluations the HQRs for the Rapid Deceleration and Race Track were good, and the only required effort was maintaining altitude and airspeed for the respective maneuvers. With the cockpit layout of the simulator, the Step Decrease rating was fair. In contrast to the airplane, the manifold pressure gauges were easy to incorporate into the instrument cross check, but single pilot workload is higher with the timed power reductions and altitude tolerances. The 4-Leg maneuver was also rated as fair, and the maneuver is not difficult to fly, however, the airspeed tolerance requires a high degree of focus. The Circle maneuver was rated highest (Level II) due to the pitch control required to maintain airspeed and altitude at a power setting established prior to entering the maneuver. Roll control required to maintain a constant bank angle and load factor added to the complexity of the maneuver. The simulator did not require power increases to maintain airspeed and altitude in any of the turns, as was needed in the actual aircraft.

The average flight times for each maneuver are summarized in Table 2 and are representative of on-condition times including turns in each of the maneuvers. The 4-Leg, Race Track, and Circle maneuvers were flown at four different airspeeds and flight times shown in the table do not include set-up time between each airspeed run. An average of two minutes could be added between airspeed runs for each of these three maneuvers which would add approximately six minutes to their total time. In comparison, the Step Decrease and Rapid Deceleration maneuvers required significantly less time when set-up time is included.

Table 2. Calibration Maneuver Times.

\begin{tabular}{|ccc|}
$\begin{array}{c}\text { Flight Test } \\
\text { Technique }\end{array}$ & $\begin{array}{c}\text { Aircraft } \\
\text { Flt Time } \\
\text { (Minutes) }\end{array}$ & $\begin{array}{c}\text { Simulator } \\
\text { Flt Time } \\
\text { (Minutes) }\end{array}$ \\
\hline Rapid Decel & $\mathbf{4}$ & $\mathbf{5}$ \\
\hline Step Decrease & $\mathbf{6}$ & 6 \\
\hline Circle & Not Flown & 6 \\
\hline Race Track & $\mathbf{1 6 . 5}$ & 15 \\
\hline GPS-4-Leg & 28 & 21 \\
\hline
\end{tabular}

\section{B. Calibration Model Structure}

A fundamental part of any system identification method is the selection of model structure and corresponding model parameters. The airspeed calibration format typically presents normalized pressure error $\left(\Delta \mathrm{p} / \mathrm{q}_{\mathrm{c}}\right)$ as a function of indicated airspeed $\left(\mathrm{V}_{\mathrm{ic}}\right.$ ). Whereas existing calibration methods compute the normalized pressure error for single airspeeds, the output error method provides a continuous model of the error as a function of indicated airspeed. For the purposes of this research, several models have been evaluated including those illustrated in Fig. 4. 


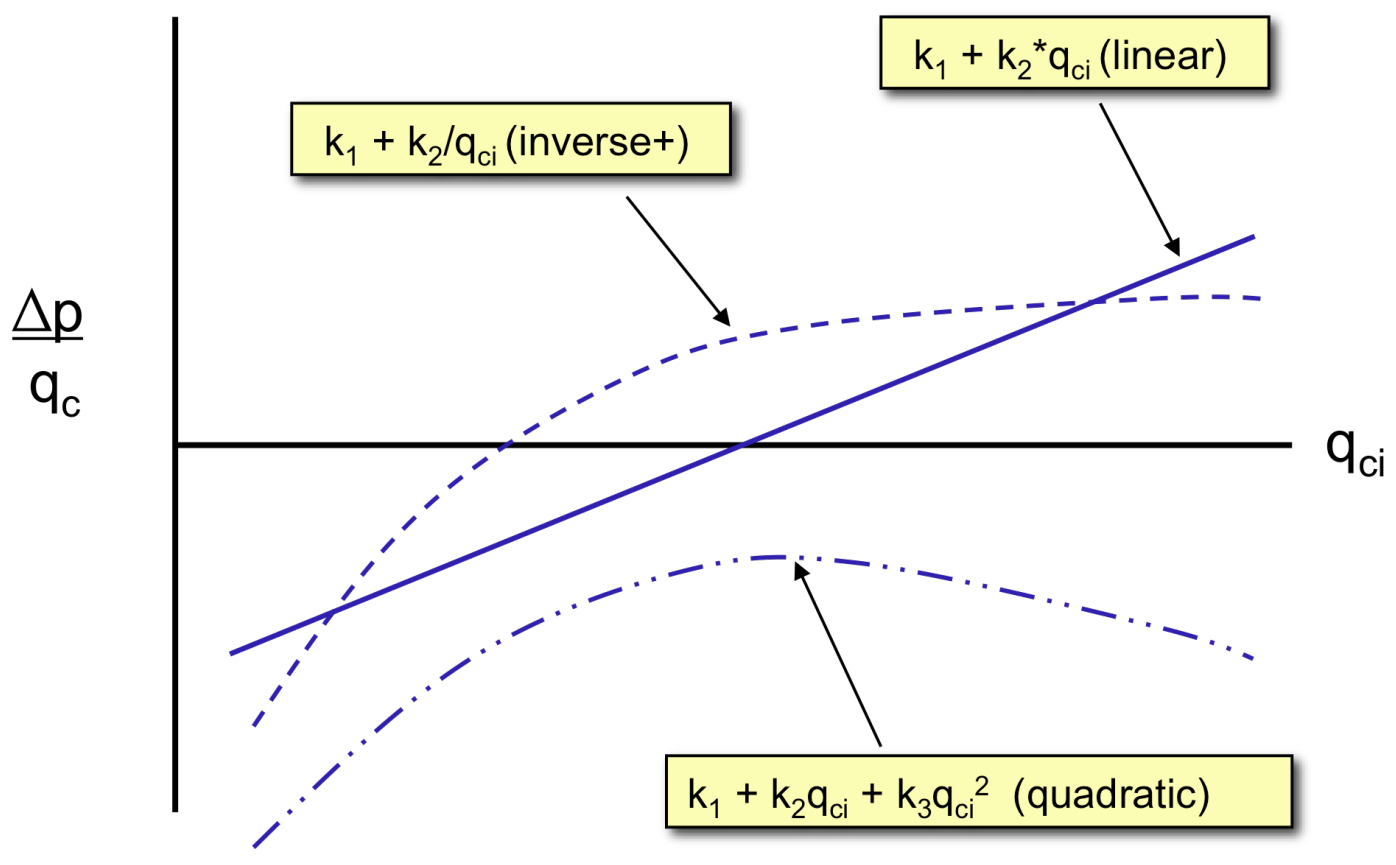

Figure 4. Illustration of Pressure Error Model Structures.

Based on commonly published flight test results, the Inverse+ and Quadratic models are considered the most likely to represent typical pressure errors for fixed wing airplanes. For the purposes of this research, selection of pressure error models was based on visual analysis of the flight data. However, it is recognized that optimal model structure can be determined using statistical-based methods but was not addressed as part of this paper.

The simulator was used to address effects of various maneuvers, data rates, and model structure on calibration accuracy. The approach involved injecting a defined pressure error and noise into the simulator airspeed calculations and then computing the calibration using several different pressure error models and different data rates. For this research, the Inverse+ model was used to corrupt the dynamic pressure values computed in the simulator, and the calibration was subsequently computed using the Quadratic, Inverse+, and Linear models along with a simple bias model. Assessment of calibration accuracy was based on the confidence bounds (2- $\sigma)$ which were computed from the residuals between the data measurements (flight and simulation) and the model.

\section{Data Acquisition System Requirements}

When discussing the DAS in this paper, a low end DAS represents mixed data rate systems with air data at 20 $\mathrm{Hz}$ or less and GPS data at $1 \mathrm{~Hz}$. These systems are typically limited to providing GPS velocity North and velocity East, and do not offer an integrated IMU/GPS solution. A high end DAS represents systems with air data and a tightly coupled IMU/GPS solution at $50 \mathrm{~Hz}$ or greater. These systems provide inertial velocity North, East, and Down. For the purposes of this paper both low and high end DAS were simulated. The low end DAS simulates the Piper Navajo's $20 \mathrm{~Hz}$ air data and $1 \mathrm{~Hz}$ GPS data capability. The high end DAS simulates $50 \mathrm{~Hz}$ and $100 \mathrm{~Hz}$ air and inertial data capabilities. All three DAS rates are representative of those used for flight test aircraft. In the following figures reference to DAS data rate is labeled as $20 / 1 \mathrm{~Hz}, 50 / 50 \mathrm{~Hz}$ and $100 / 100 \mathrm{~Hz}$ where the first value is that for air data and the second value is that for GPS data.

The simulator was used to study the effects of data rate, data density, maneuver variations, and inclusion of GPS vertical speed on overall accuracy. In order to simulate system noise and sensor errors, the simulator dynamic pressure was corrupted by injecting known white random noise in addition to simulated pressure errors. The injected noise was an order of magnitude higher than the specification sheet for the actual flight instruments. Even when the plumbing and fixtures are considered, the simulated dynamic pressure errors were still considered to be conservative.

While there is no criteria for data density using system identification methods such as OE, it is generally expected that a uniform data distribution will minimize confidence bounds of identified model parameters. Figure 5 represents comparisons of the data density and lumped distributions for Step Decrease and 4-Leg maneuvers. To obtain more uniform data distributions, the 4-Leg maneuver (with four and six airspeeds), and the Step Decrease 
maneuver (with the time for power reductions varied) are compared. The purpose of this trial was to determine if sample size and/or a more uniform data distribution affected the 2- $\sigma$ values. The corresponding results of this test are illustrated in Fig. 6. There was a modest decrease in 2- $\sigma$ values for the 4-Leg maneuver with the increased sample size (due to additional airspeeds). Conversely, the Step Decrease method showed a slight increase in 2- $\sigma$ values with the larger sample size. It is noteworthy that the 2- $\sigma$ values were smaller for the Step Decrease maneuver although the amount of data points in the Step Decrease were about one-third that of the 4-Leg.
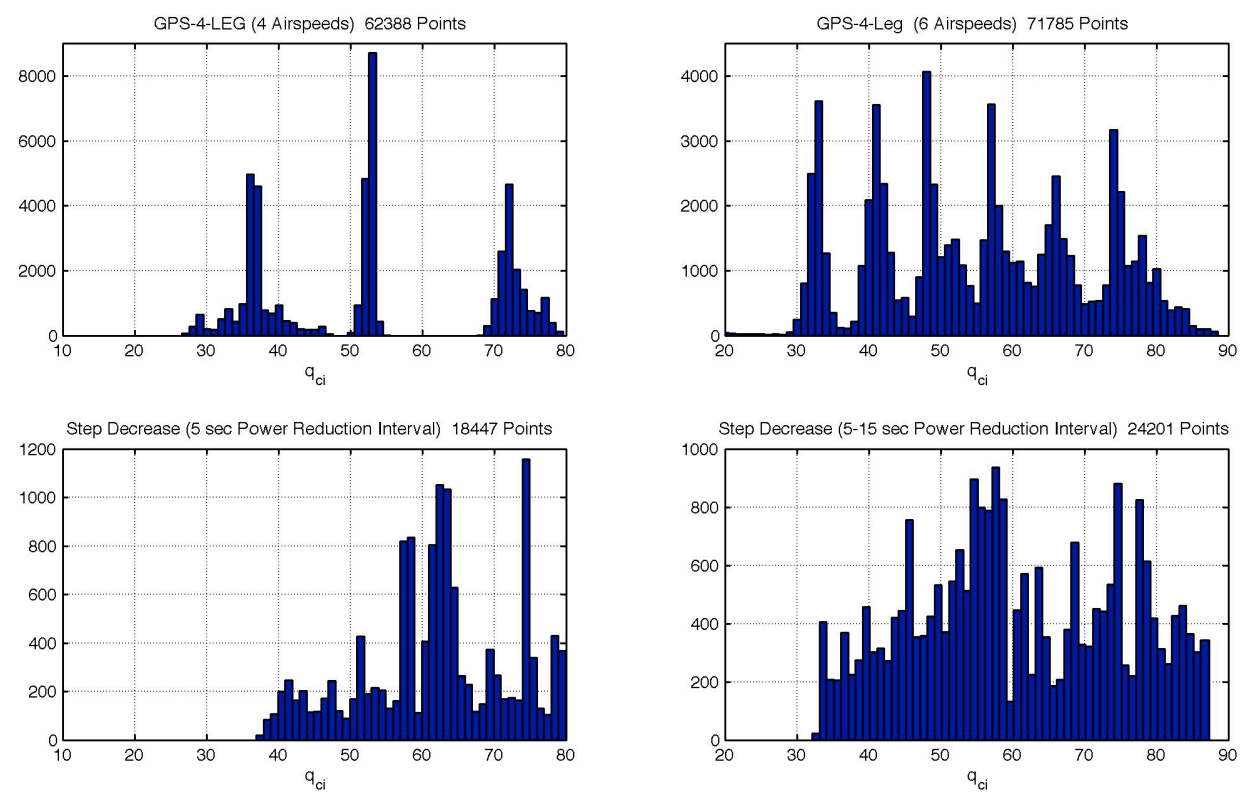

Figure 5. Data Density and Distribution (50/50 Hz Simulator Data, Corrupted Inverse+ and Estimated Inverse+)

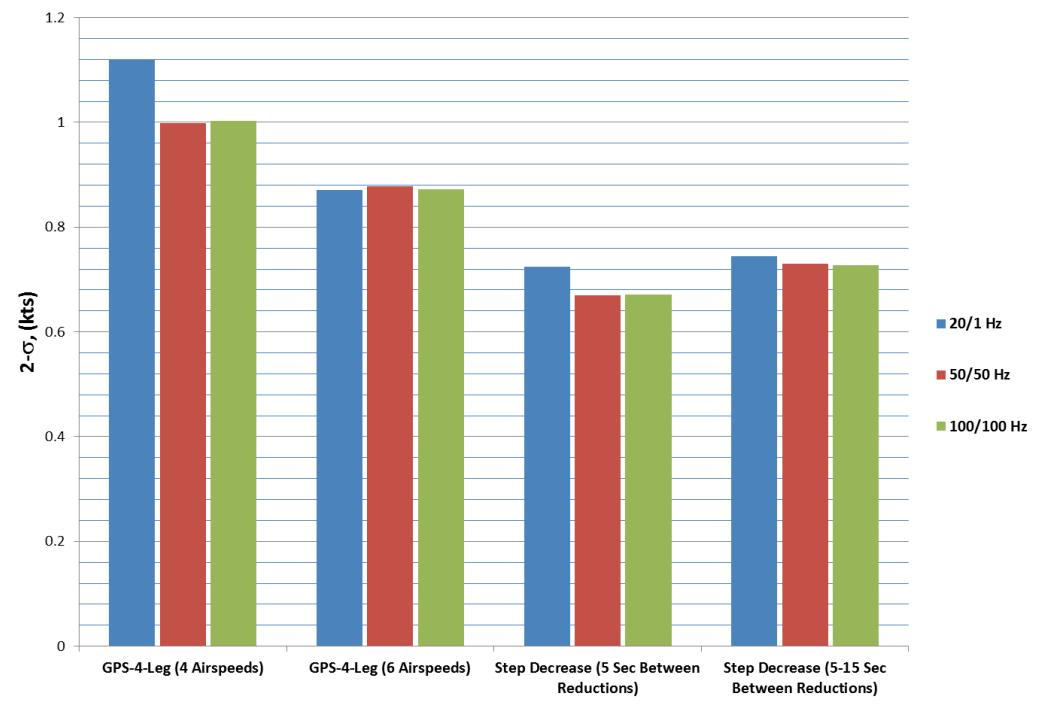

Figure 6. Effects of Data Density on 2- $\sigma$. (Simulator Data, Corrupted Inverse+ and Estimated Inverse+) 
Initial simulator testing showed significant sensitivity to turbulence and wind vector variations. Turbulence introduces noise into the system identification problem. Noise has been shown to degrade the accuracy and convergent capability of output error ${ }^{5-6}$. Additionally, wind vector variations violate the algorithm assumption that the wind vector remains constant throughout the maneuver. How much wind speed and direction variations can be tolerated is a topic of ongoing research.

The inclusion of inertial vertical velocity compensation was also evaluated to determine its effects on the 2- $\sigma$ values. Figure 7 represents the addition of vertical velocities on the 4-Leg and Step Decrease maneuvers. Both maneuvers showed a decrease in 2- $\sigma$ values where the 4-Leg maneuver had the largest 2- $\sigma$ reduction. The sensitivity of 4-Leg data to inertial vertical velocity is in large part due to altitude variations that are part of holding a test point for tens of seconds. It is also observed that the smallest 2- $\sigma$ reduction occurs for the lowest sample rates. This can be explained by the effects of multi-rate data. Additional error is introduced by using $1 \mathrm{hz}$ data (similar to sample and hold) with $20 \mathrm{hz}$ data, which offsets the improvement due to vertical velocity compensation.

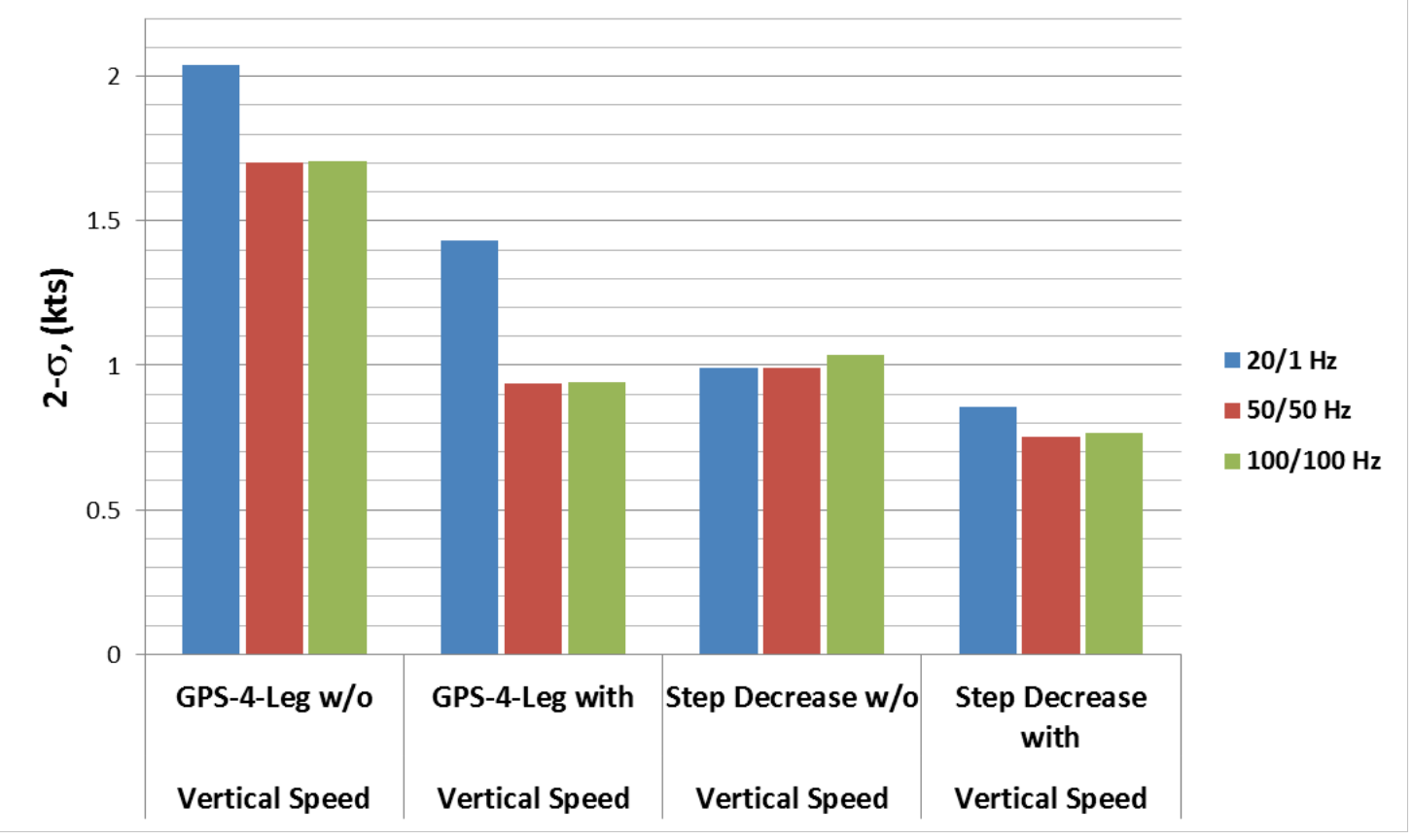

Figure 7. Effects of Vertical GPS Velocity on 2- $\sigma$. (Simulator Data, Corrupted Inverse+ and Estimated Inverse+)

An evaluation was performed to determine if the on-condition data or the full data set (turn data included) should be used for the maneuvers. A comparison was then made with the inclusion of inertial vertical velocity to examine its effects on the 2- $\sigma$ values. Figure 8 shows these comparisons for the Step Decrease maneuver for corrupted and estimated Inverse+ pressure model. These comparisons show that the full data set and the oncondition data without vertical velocity were comparable. However, with the inclusion of vertical velocity, the 2- $\sigma$ values decreased by as much as $25 \%$. The $2-\sigma$ values for the full data set and on-condition data with vertical velocities are virtually identical. This result represents the robustness of the system identification method to the inclusion or exclusion of turn data. 


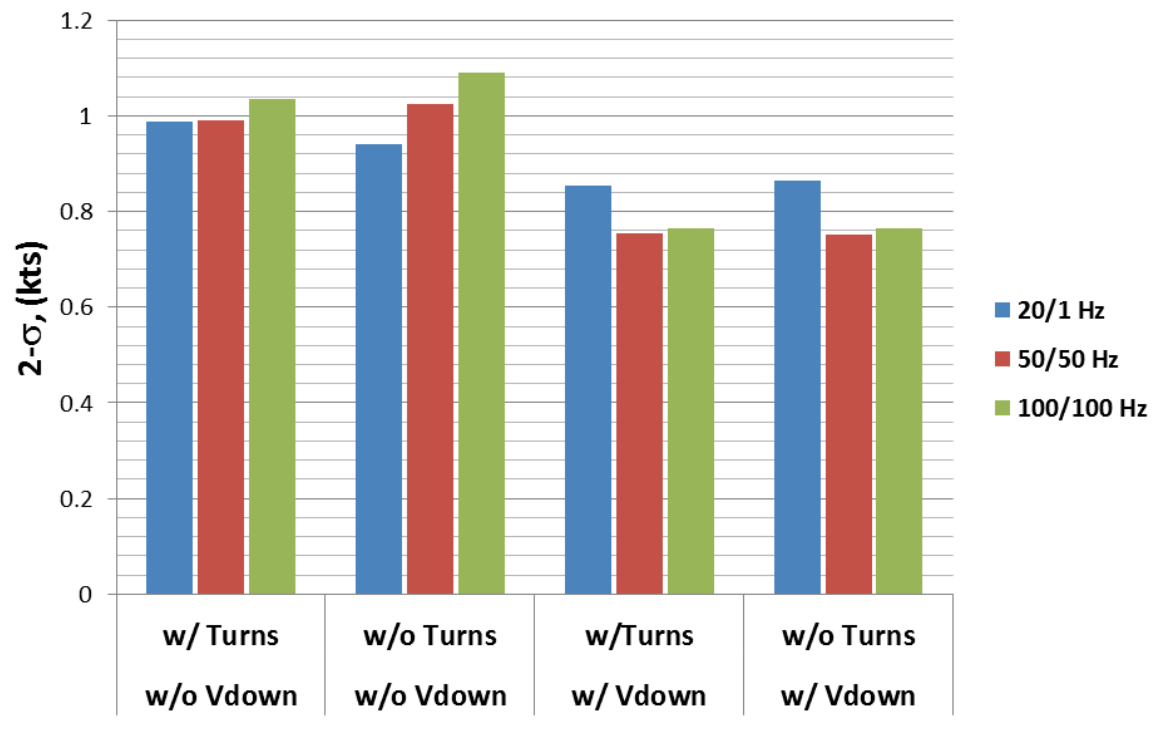

Figure 8. Effects of Turn Data and with/without GPS Vertical Velocity on 2- $\sigma$. (Step Decrease Method, Simulator Data Corrupted Inverse+ and Estimated Inverse+)

\section{Effects of Model Structure on Calibration Accuracy}

Figure 9 depicts the calibration accuracy for various model structures and maneuvers at the $50 / 50 \mathrm{~Hz}$ rate. All of the data was corrupted with an Inverse+ pressure model and modeled using Quadratic, Linear, Inverse +, and Bias pressure models. This data rate was charted because it is representative of the trends exhibited by the three data rates used. The Quadratic and Inverse+ pressure model values are almost identical for the five maneuver types, and the Inverse+ pressure model was expected to be the best fit since the data was corrupted with an Inverse+ pressure model. The Bias pressure model under parameterizes the data and the 2- $\sigma$ values were double that of the other three. Hence, the Bias model is not a viable solution for typical pressure error calibration.

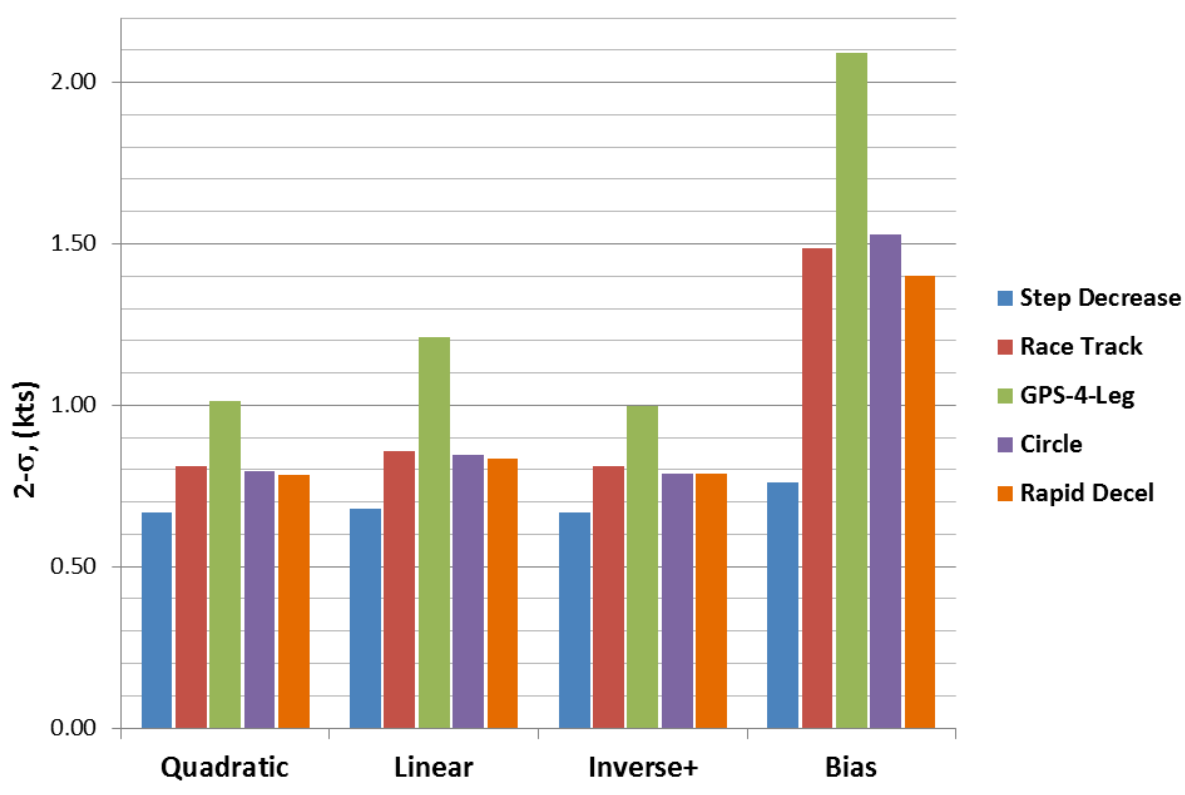

Figure 9. Effect of Model Structure On Confidence Bounds.

(Data Rate: 50/50 Hz; Simulator Data Corrupted Using Inverse+ Model)

American Institute of Aeronautics and Astronautics 


\section{E. Effects of Maneuver on Calibration Accuracy}

Both low and high end DAS were to simulate flight data for the five maneuver types. This was done to determine if there is an optimum data update rate and to see what the sensitivity is for each maneuver type. The chart in Fig 10 shows that there is no significant variation between the $50 / 50 \mathrm{~Hz}$ and $100 / 100 \mathrm{~Hz}$ data. The higher data update rate did not yield any appreciable benefit to the 2- $\sigma$ values and illustrates that more sophisticated equipment is not required. Data taken at the $20 / 1 \mathrm{~Hz}$ rate on average increased the $2-\sigma$ values by approximately $10 \%$, hence showing that this is also an acceptable data update rate for these maneuvers.

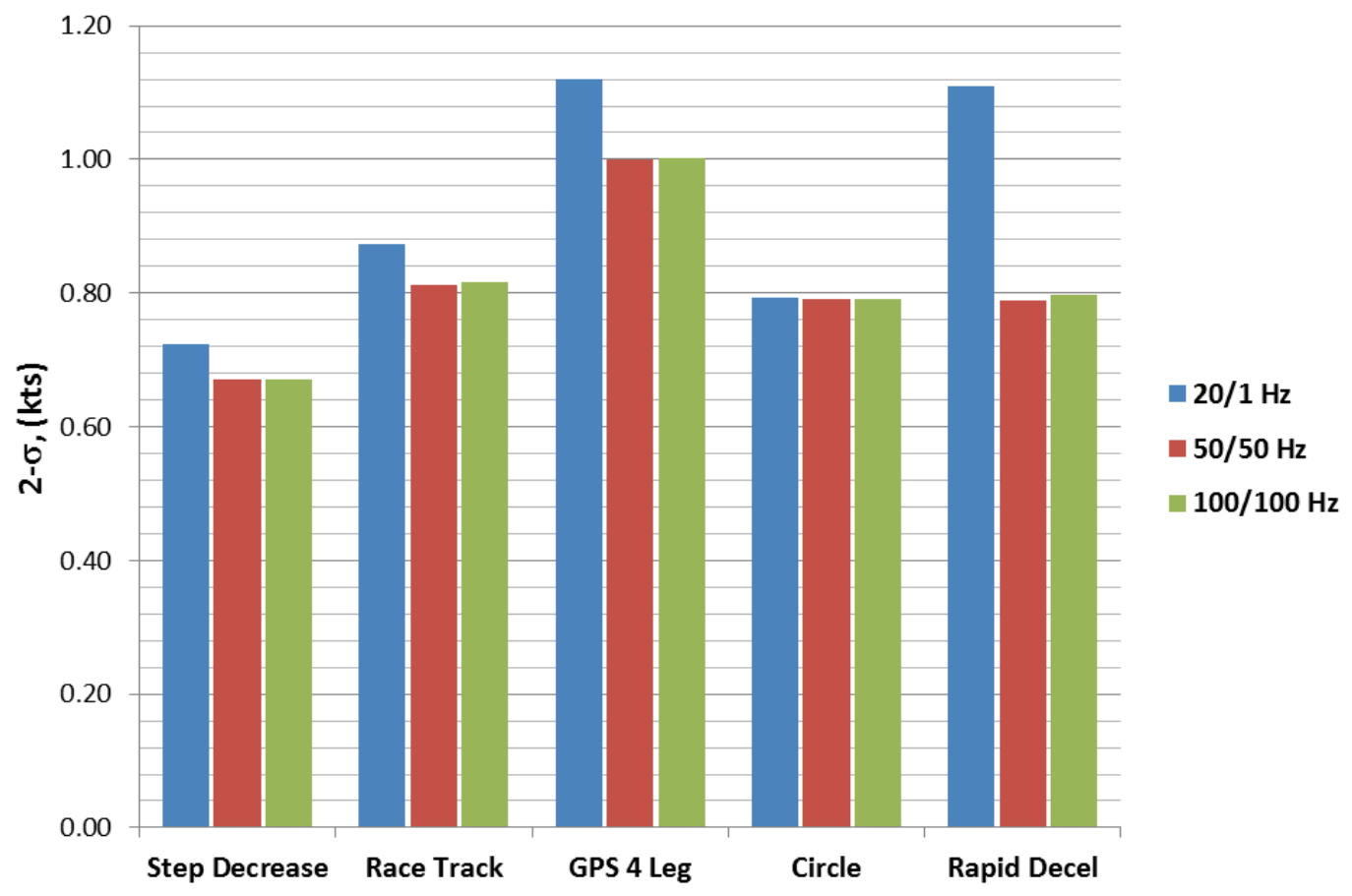

Figure 10. Effect of Maneuver and Data Frequency On Confidence Bounds. (Simulator Data, Corrupted With Inverse+ Model, Estimated Using Inverse+ Model)

The data from Figs. 9 and 10 show that the Step Decrease method produces the lowest 2- $\sigma$ values. Also, Table II reveals that the Step Decrease method involves the least flight time to perform. The Circle, Rapid Deceleration, Race Track, and 4-Leg respectively follow in 2- $\sigma$ results.

\section{F. Flight Calibration Results}

Airspeed calibration results for the Navajo are shown in Figs. 11 through 14 for the flaps up/gear up configuration with the pod on. Based on analysis of the flight results, inclusion of data acquired during turns increased 2- $\sigma$ values, so only data for straight legs was included in the calibrations. It is noted that the gap in data was due to inadvertent loss in airspeed during the turn. In addition, the quadratic model structure was used for all calibrations as this was shown to provide the best overall calibration results based on the $2-\sigma$ errors . As a reference, the maximum allowable airspeed error based on FAR 25.1323 is shown in the figures. 

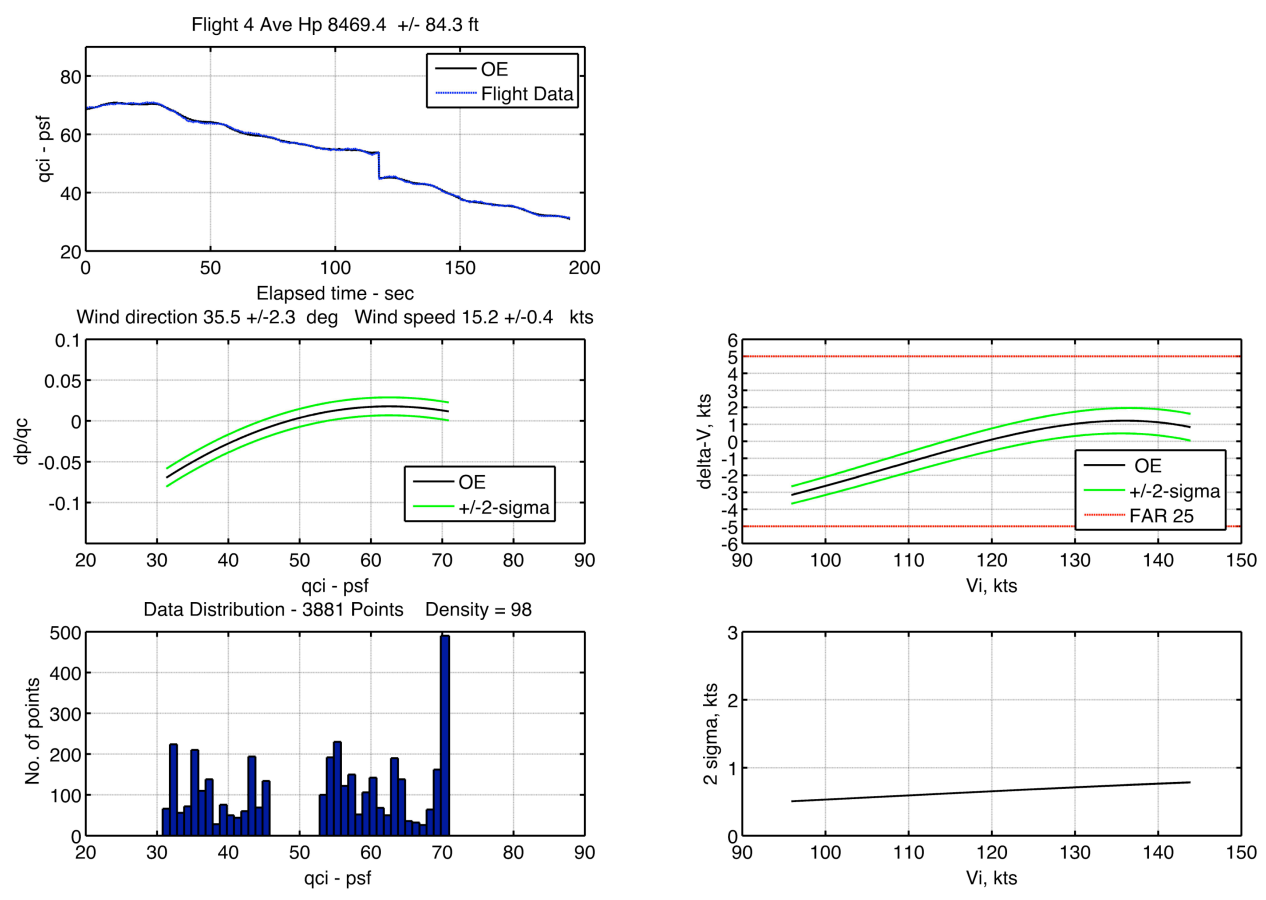

Figure 11. Airspeed Calibration Results, Pod On, Gear/Flaps Up, Step Decrease Maneuver.
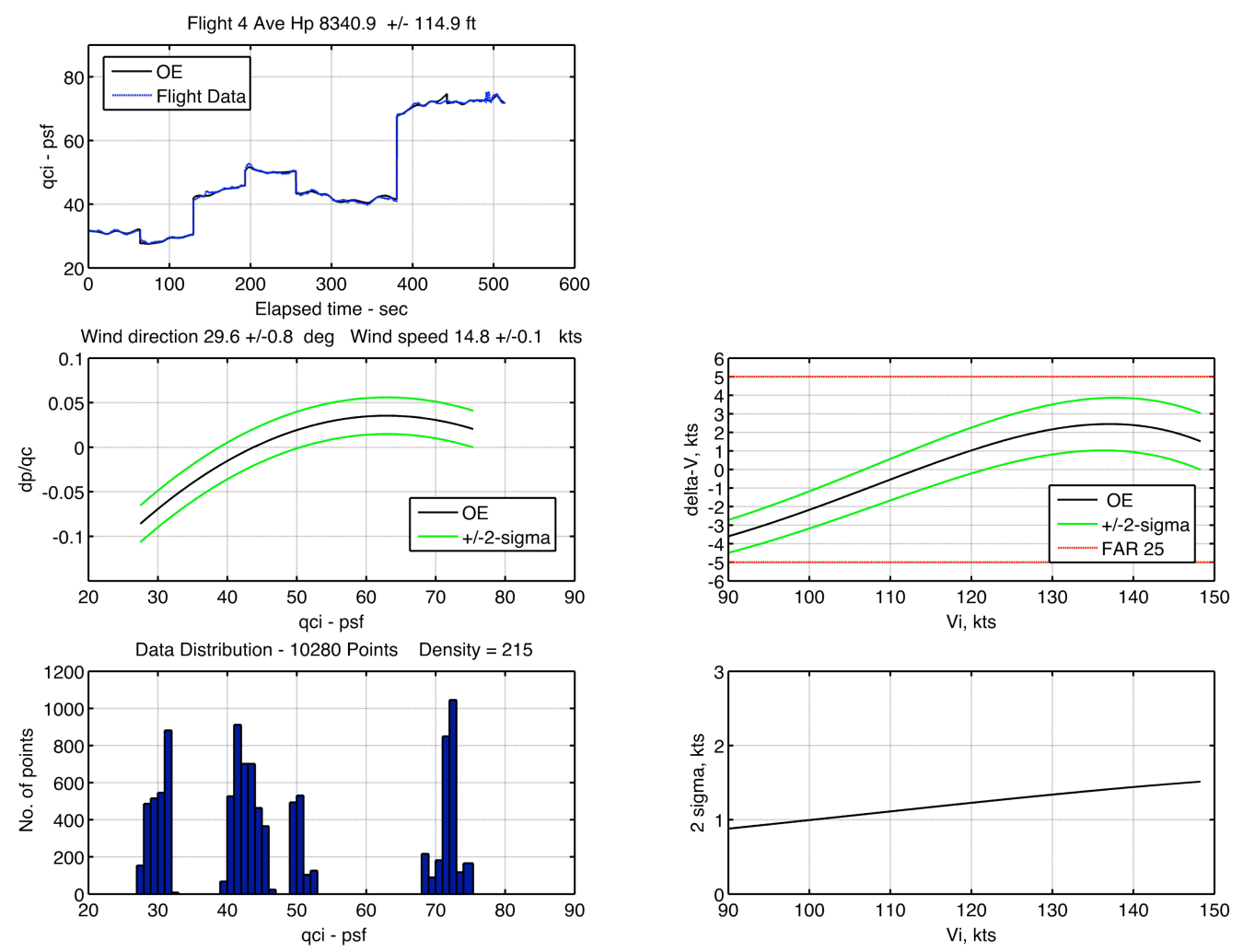

Figure 12. Airspeed Calibration Results, Pod On, Gear/Flaps Up, Racetrack Maneuver. 

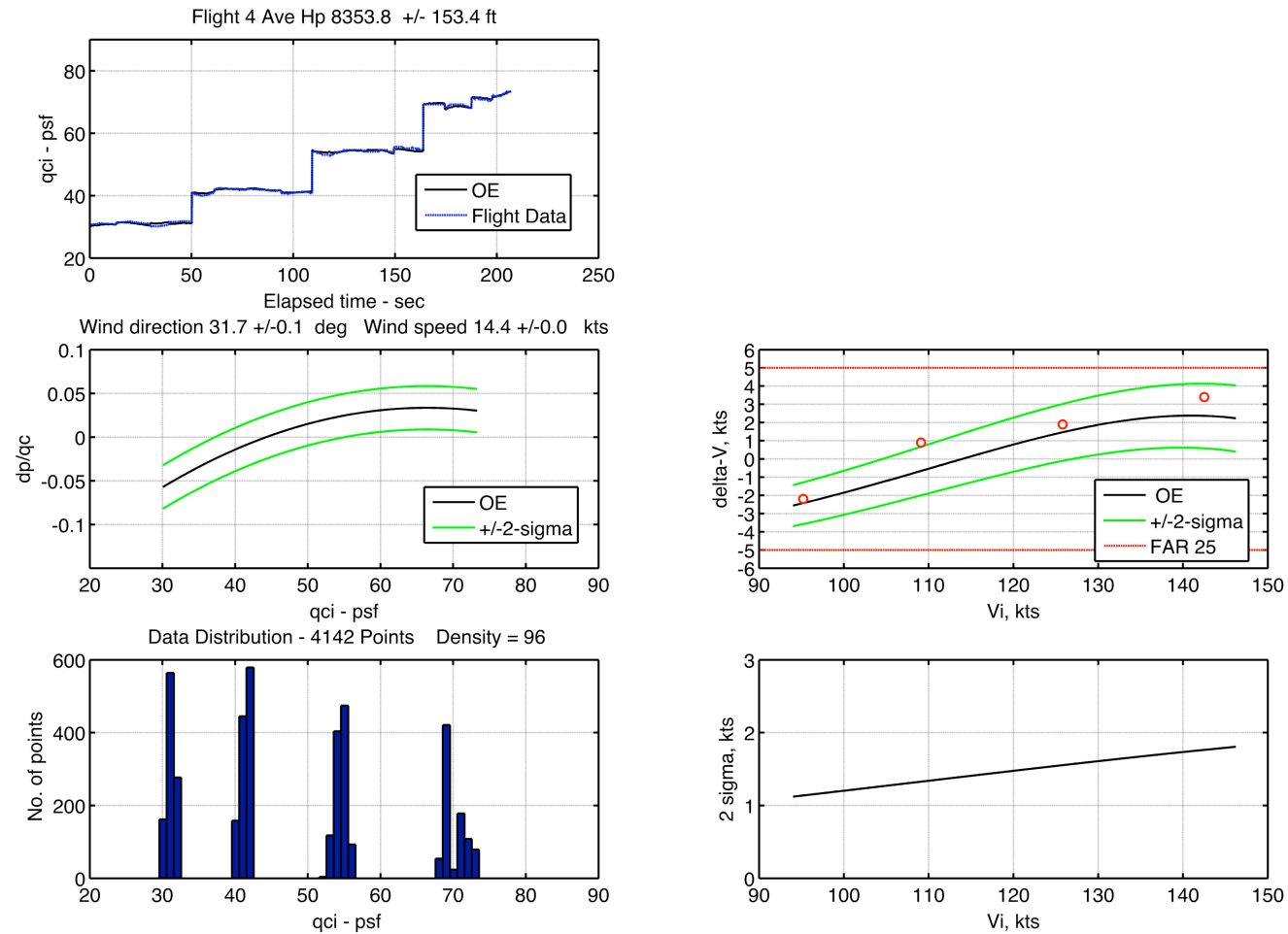

Figure 13. Airspeed Calibration Results, Pod On, Gear/Flaps Up, 4-Leg Maneuver. (Red circular symbols denote calibration for fixed airspeed method)
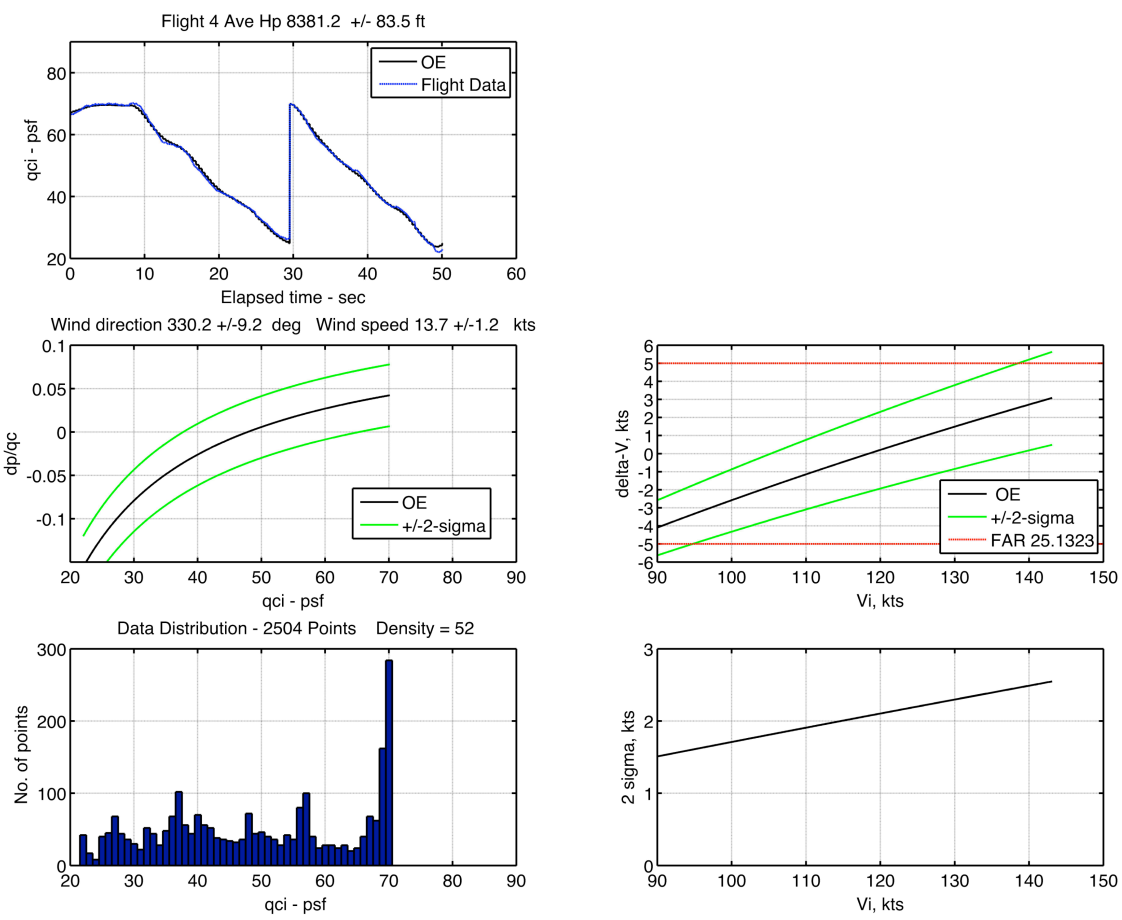

Figure 14. Airspeed Calibration Results, Pod On, Gear/Flaps Up, Rapid Decel Maneuver. 
By inspection of Figs. 11 through 14 and the summary provided in Table III, several important conclusions can be drawn. First, all methods yield similar results for the mean calibration curve, however there were significant differences in accuracy and flight time. The Step Decrease maneuver provided the highest accuracy and shortest test time for the calibration. However as previously discussed, this maneuver exhibited the highest pilot workload. The lowest calibration accuracy was provided by the Rapid Decel, probably due to the small sample size and rapid change in airspeed. Figure 13 shows a comparison of the OE-derived calibration to the conventional calibration (red symbols) for fixed airspeeds for the 4-Leg method. This result shows good agreement, as expected, although confidence bounds are typically not included with the latter approach.

Table III. Summary of Flight Calibration Results.

\begin{tabular}{|l|c|}
\hline \multicolumn{1}{|c|}{ Maneuver } & $\begin{array}{c}\text { Average 2- } \sigma \text { Error } \\
\text { KCAS }\end{array}$ \\
\hline Step Decrease & 0.75 \\
\hline Racetrack & 1.2 \\
\hline 4-Leg & 1.5 \\
\hline Rapid Decel & 2.1 \\
\hline
\end{tabular}

\section{Summary and Conclusions}

The purpose of this research was to investigate the potential for a viable alternative to the conventional FAA Part 23 and Part 25 airspeed calibration methods (i.e. trailing cone) and to the GPS-4-Leg maneuver. A goal was to develop an accurate technique that enables reductions in test equipment and flight time, thus reducing costs. The approach involved development of flight maneuvers, analysis of data acquisition requirements, and analysis of pressure error models based on system identification methods.

A GPS-based inflight pitot-static calibration method, originally developed for the NASA AirSTAR subscale flight test facility, was tested utilizing an instrumented Piper Navajo research aircraft. In addition, an engineering flight simulator was used to investigate test maneuver requirements and handling qualities issues for this scale of aircraft. A total of five airspeed calibration maneuvers were studied: 4-Leg, Racetrack, Step Decrease, Rapid Deceleration, and Circle. All five were tested in the engineering simulator, and all except the Circle maneuver were flown in the Piper Navajo.

Calibration maneuvers were evaluated in flight and in the simulator comparing the flight time required for each method. Use of the simulator to compare maneuver handling qualities and flight times to those in the aircraft show how each maneuver equates to the current accepted methods. Also, the simulator aided in verifying the time required to perform each maneuver as well as understanding pilot workload issues. These results showed that the Step Decrease method can be accomplished in approximately one-fourth the time as the current 4-Leg maneuver. The three additional maneuver techniques tested also required less time than the 4-Leg. This reduction in flight time would lead to cost savings. The Step Decrease 2- $\sigma$ values attained in the simulator and flight represent the best results of the tested maneuvers. Although the GPS-4-Leg method is currently accepted as a standard method, the three other maneuvers tested produced results that can potentially provide more accurate airspeed calibration.

Pilot workload for the Step Decrease and the Circle maneuver was higher than the 4-Leg maneuver due to the increased effort for instrument cross-check and difficulty in performing the flight maneuver, as indicated by the increase in handling qualities ratings. The Step Decrease maneuver required power manipulation and manifold pressure gauge readings to be of primary focus, followed by heading. The Circle maneuver added complexity by requiring a constant bank angle, airspeed, and altitude simultaneously, which increased workload. These factors were not significant enough to outweigh the benefits of improved calibration accuracy (lower 2- $\sigma$ values) and reduced flight times.

Comparisons of the three data rates $(100 / 100 \mathrm{~Hz}, 50 / 50 \mathrm{~Hz}, 20 / 1 \mathrm{~Hz})$ showed an improvement in 2- $\sigma$ values for $50 / 50 \mathrm{~Hz}$ over $20 / 1 \mathrm{~Hz}$ but no appreciable change between $100 / 100 \mathrm{~Hz}$ and $50 / 50 \mathrm{~Hz}$ data rate systems. Significant 2- $\sigma$ improvement can be obtained through hardware capable of calculating vertical GPS velocity, such as a coupled IMU/GPS unit.

Recommendations for future work on this subject include effects of variable winds, effects of sideslip, and broader sampling rate effects on 2- $\sigma$ values. In addition, an analytical approach should be used to determine model structure that provides the best model fit to the measured data. 


\section{References}

${ }^{1}$ Lewis, Gregory W., "Using GPS to Determine Pitot-Static Errors", National Test Pilot School, 14 August 2003.

${ }^{2}$ Foster, John V. and Cunningham, Kevin, "A GPS-Based Pitot-Static Calibration Method Using Global Output-Error Optimization, ," AIAA-2010-1350, presented at 48 ${ }^{\text {th }}$ AIAA Aerospace Sciences Meeting, January 2010.

${ }^{3}$ Klein, Vladislav and Morelli, Eugene A., Aircraft System Identification, Theory and Practice, AIAA Education Series, 2006.

${ }^{4}$ Cooper, George E. and Harper, Robert P. Jr., "The Use of Pilot Rating In the Evaluation of Aircraft Handling Qualities", NASA TN-5153, April 1969.

${ }^{5}$ Maine, R.E. and Iliff, K.W., "Formulation and Implementation of a Practical Algorithm for Parameter Estimation with Process and Measurement Noise," SIAM Journal of Applied Mathematics, Vol. 41, No. 3, 1981, pp. 558-579.

6Maine, R.E. and Iliff, K.W., "Identification of Dynamic Systems,” AGARD AG-300 Vol. 2, Jan. 1985. 